\title{
NEURO-FUZZY MODELLING OF BLENDING PROCESS IN CEMENT PLANT
}

\author{
Dauda Olarotimi Araromi', Stephen Ayodele Odewale', Jimoh Olugbenga Hamed ${ }^{2}$ \\ 1 Department of Chemical Engineering, Lautech, PMB 4000, Ogbomoso, Nigeria, e-mail: aradachem@yahoo. \\ com, stephenodewale@yahoo.com \\ 2 National Space Research and Development Agency, Nigeria, e-mail: hamedjimoh45@yahoo.com
}

Received: 2015.09.08

Accepted: 2015.11.14

Published: 2015.12.04

\begin{abstract}
The profitability of a cement plant depends largely on the efficient operation of the blending stage, therefore, there is a need to control the process at the blending stage in order to maintain the chemical composition of the raw mix near or at the desired value with minimum variance despite variation in the raw material composition. In this work, neuro-fuzzy model is developed for a dynamic behaviour of the system to predict the total carbonate content in the raw mix at different clay feed rates. The data used for parameter estimation and model validation was obtained from one of the cement plants in Nigeria. The data was pre-processed to remove outliers and filtered using smoothening technique in order to reveal its dynamic nature. Autoregressive exogenous (ARX) model was developed for comparison purpose. ARX model gave high root mean square error (RMSE) of 5.408 and 4.0199 for training and validation respectively. Poor fit resulting from ARX model is an indication of nonlinear nature of the process. However, both visual and statistical analyses on neuro-fuzzy (ANFIS) model gave a far better result. RMSE of training and validation are 0.28167 and 0.7436 respectively, and the sum of square error (SSE) and R-square are 39.6692 and 0.9969 respectively. All these are an indication of good performance of ANFIS model. This model can be used for control design of the process.
\end{abstract}

Keywords: neuro-fuzzy, blending, carbonate content, raw mix.

\section{INTRODUCTION}

There has been tremendous growth in the cement industry during the past century. Humans had earlier discovered certain natural rocks which, gave a product that hardened on the addition of water through simple calcinations. Yet, real advances did not come until physiochemical studies and chemical engineering laid the basis for modern efficient plants working under closely controlled conditions with a variety of raw materials [6].

With cement demand at its highest level ever, cement plants are continually being pushed to the limit. A lot is being demanded of both personnel and equipment, not only to maintain production, but also to establish new records without sacrificing quality. These therefore pose challenges to cement manufacturers as they strive for consistent quality products while reducing input costs.
Many of these challenges can be addressed through effective control of the cement plant from the raw material stage down to the finished product stage. One of the stages involved in cement production is blending raw materials to obtain raw mix for clinker production. This stage must be well monitored in other to control the carbonate content of the raw mix. The total carbonates content of the raw mix is important as it determines the lime composition of the raw mix and the loss on ignition of the raw mix in the kiln during sintering.

The raw materials, most importantly limestone, is usually quarried from local rock, which most often varies in concentration due to the presence of impurities in the limestone, therefore, clay feeding into the limestone is carried out to regulate the carbonate composition of the raw mill output. In many plants, the raw materials are 
roughly blended in a prehomogenization pile before grinding in a raw mill to a raw mix [6]. The total carbonate content of the raw mix is an important composition that must be controlled and put on check to ensure that they are within the required specification [12]. In order to study the behavior and control the blending process, we need a mathematical representation of the physical and chemical phenomena taking place in the process.

Many works have been conducted on modelling the blending processes. Three different linear multivariable stochastic Autoregressive exogenous (ARX) models were proposed to predict iron oxide and/or lime module of the raw mix [8]. A stochastic multivariable dynamic model was developed and a model predictive controller was designed to calculate the optimal feed ratios of the raw materials [13]. LU (Cholesky) was used for decomposition of the covariance matrix in multivariate geostatistical simulation to obtain the chemical composition signal in order to produce an output stream with cement characteristics as close as possible to desired targets [5]. Many of these modelling approaches are linear and these produce models that can only operate in a narrow operating range, however, most of the chemical processes, if not all, are inherently nonlinear. In order to meet stringent environmental regulation, competitiveness in the price of product and demand for high quality product often require operating process plant at wide operating conditions, it is essential to have the process represented in a nonlinear modelling system [2].

A new mathematical modeling technique, known as soft computing, is promising in modeling complex nonlinear system. Soft computing, a practical alternative for solving computationally complex and mathematically intractable problems, consists of fuzzy inference systems, neural networks, chaos theory and genetic algorithms [4]. Two or more of these techniques have been combined to handle real time problems in order to achieve the level of accuracy and efficiency needed in practice. One of these hybrid techniques is a neuro-fuzzy scheme $[7,9,11,10,1]$. A neurofuzzy system is a fuzzy inference system framed in a network structure, and reinforced with neural network learning abilities [4]. The learning ability of neural networks have been employed to cope with the difficulties arising from the human-based construction of fuzzy by automating and supporting the process of developing a fuzzy system for a given application [3]. Neuro-fuzzy systems have found a wide success due to their hybrid nature which allows the acquisition of accurate knowledge from numerical data via neural learning, with the ability of representing it in a fuzzy rulebased structure so as to be classified as human readable [1].

In this work, dynamic model of blending process in cement plant was carried out using input-output data collected from one of the cement plants in Nigeria. Neurofuzzy system which is based on the adaptive network-based fuzzy inference system (ANFIS) was employed in an attempt to predict dynamic blending process in cement plant. The input is clay feed rate and output is the total carbonate content of the raw mix. The flow of limestone is kept constant but with varying composition due to the presence of impurities.

\section{PROCESS DESCRIPTION}

The production of cement involves broadly four stages: extraction and pre-processing of raw materials; pyroprocessing to produce clinker; blending and grinding of clinker to cement; storage, packing and delivery of cement. The process for cement production is shown in Figure 1, but the concern of this work is the blending stage of the process. The important raw materials used in production of cement are limestone and clay. The limestone contain calcium carbonate which breaks down to $\mathrm{CaO}$ (an important composition of the cement) and $\mathrm{CO}_{2}$ on heating in the kiln. Clay, which contains basically silicon oxide, is used to regulate carbonate composition of the rawmix. The clay is fed into the silo at different rate depending on the total carbonate value of the limestone already present in the silo, which varies in composition due to the presence of impurity in the limestone. This is then transferred into the mill for final blending. The raw mix must be formulated to a very tight chemical specification. Typically, the content of individual components in the rawmix must be controlled within $0.1 \%$ or better. The relative amounts of each raw material must be kept constant in order to maintain steady conditions in the kiln, and to maintain constant product properties. In practice, the rawmix is controlled by frequent chemical analysis (hourly by X-Ray fluorescence analysis, or every three minutes by prompt gamma neutron activation analysis).

For this work, total carbonate (TC) values of the raw mix were obtained from TC control ti- 


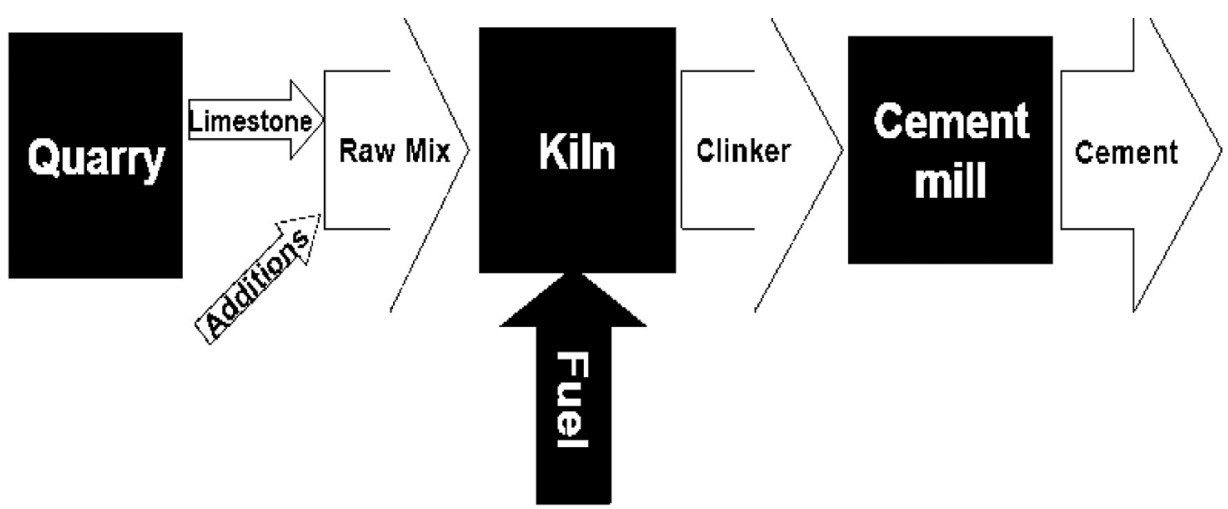

Fig. 1. Process path for cement production

tration performed every thirty minute, the average computed was compared with the standard specification to determine if the TC is high or low. When TC is high, clay feeding is increased to bring down the TC to the desired range and vice versa. The standard TC percentage is 65 $66 \%[12]$.

\section{NEURO-FUZZY NETWORK ARCHITECTURE}

A five layer network in the frame of Sugeno type model can be used to implement ANFI network. The architecture is shown in Figure 2. For two inputs $\mathrm{x}_{1}$ and $\mathrm{x}_{2}$ with two rules and one output y. The fuzzy logic inference system for identification of Sugeno type model can then be implemented as:

$$
\begin{aligned}
& \text { IF } x_{1}=A_{1} \text { AND } x_{2}=B 1 \text { THEN } y=a x_{1}+b x_{2}+c \\
& \text { IF } x_{1}=A_{2} \text { AND } x_{2}=B_{2} \text { THEN } y=a x_{1}+b x_{2}+c
\end{aligned}
$$

Figure 2 represents a Takagi-Sugeno-type of MISO neuro-fuzzy network (ANFIS), The ANFIS consists of adaptive nodes represented as squares and fixed nodes represented as circles. Using a learning rule the parameters in these nodes are adjusted to minimize a specified error measure. The first layer is used to generate the membership grades for each set of input data vectors. In this layer, each neuron is correspondent to a linguistic label. The crisp inputs $x_{1}$ and $x_{2}$ are fuzzified by

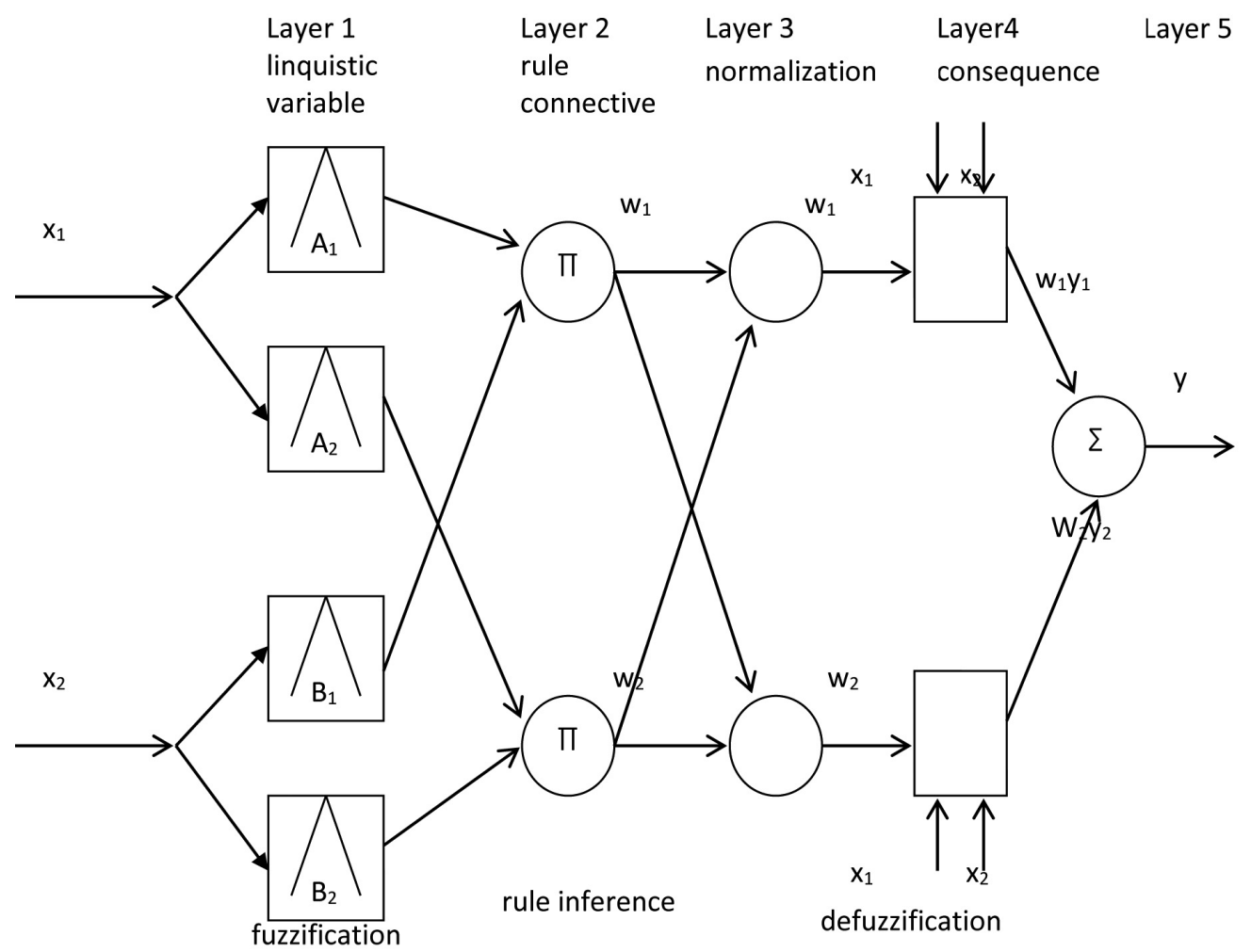

Fig. 2. Nuerofuzzy network structure 
using membership functions of the linguistic variables $A i$ and $B i$. Any of triangular, trapezoid or Gaussian membership curves can be used. In this work, Gaussian membership curve is employed and is given as

$$
\boldsymbol{\mu}=\exp \left(-\frac{1}{2}\left(\frac{x_{j}-c_{j}^{i_{j}}}{\sigma_{j}^{i}}\right)^{2}\right)
$$

where: $c_{j}^{i}, \sigma_{j}^{i}$ is the parameter set which is used to alter the shape of the membership function.

The second layer of ANFIS calculates the firing strengths of the rules as a product of the membership grades: Each node in the second layer corresponds to one fuzzy IF ... THEN rule. Each rule node performs a connective operation between rule antecedents (the premise or IF part). Usually the minimum or dot product is used as AND intersection. The union or OR is usually performed by using the maximum operator. The firing strength of the fuzzy rules can be computed as a product of the membership grades according to:

$$
\begin{gathered}
w_{i}=\min \left\{\mu_{A_{i}}\left(x_{1}, \mu_{B_{j}}\left(x_{2}\right)\right\}, i=1,2\right. \\
w_{i}=\mu_{A_{i}}\left(x_{1} * \mu_{B_{j}}\left(x_{2}\right), i=1,2\right.
\end{gathered}
$$

In layer 3 , the normalized firing strengths of the rules (i.e. the ratio of the i-th rules firing strength to the sum of all rules' firing strengths). The firing strengths $w_{\mathrm{i}}$ of the fuzzy rules are normalized according to:

$$
\bar{w}_{i}=\frac{w_{i}}{\sum_{i=1}^{2} w_{i}}
$$

Layer 4 yields the parameters of the consequent part of the rule. In this layer, every node is characterized by a function of the form:

$$
y_{i}=\bar{w}_{i}\left(a_{i} x_{1}+c_{i}\right)
$$

The fifth layer calculates the overall output as the sum of the contributions of each rule, i.e.

$$
\boldsymbol{y}=\sum_{i=1}^{2} \boldsymbol{y}_{i}
$$

In summary, the first and fourth layers in the ANFIS are adaptive layers. The first layer has two turn able parameters $c_{j}^{i}, \sigma_{j}^{i}$ which characterize the shape of the input membership functions. These parameters are associated with the IF part of the rule (the so-called premise parameters). The fourth layer also has three adjustable parameters, $[\mathrm{a} i, \mathrm{~b} i, \mathrm{c} i]$, the consequent parameters, which are associated with the THEN part of the rule.

\section{DATA PREPROCESSING}

The input variable $u(k)$ is the clay feed rate used as a correction factor to stabilize the concentration of limestone; the output variable $y(k)$ is the results of laboratory analysis conducted to determine the total carbonate content of the raw mix which in turn influence the oxide composition. Nine hundred and sixty-six input-output data points were collected from the process at a sampling time of 0.2 minutes.

In practice, data preprocessing is necessary in order to eliminate outliers by sorting out the "good" data from the "bad." This preliminary step ensures meaningful conclusions in subsequent parts of the analysis. Outliers are data values that are dramatically different from patterns in the rest of the data which may be due to measurement error, these were identified by looking out for values more than a certain number specifically two times the standard deviations $(\sigma)$ from the mean $(\mu)$ of the raw data. The data was then smoothened using the convolution function to reveal its main dynamic features before it is used for model identification. The extent of the smoothing was controlled with the variable span. The first hundred processed data set is shown in Figure 3.

\section{Model identification and validation}

In addition to the ANFIS approach, AutoRegressive eXogenous(ARX) approach was also utilized to model the plant and a comparison was carried out to justify the performance of the ANFIS model.

A conventional method is to remove the means from the data and assume a linear model of the form:

$$
\begin{aligned}
& y(k)+a 1 * y(k-1)+\ldots+a m * y(k-m)= \\
& =b 1 * u(k-d)+\ldots+b n * u(k-d-n+1)
\end{aligned}
$$

To find an ARX and ANFIS model for the blending process, the data set was divided into a training (500) and a checking (410) data set. For ARX, an exhaustive search was performed to find the best combination of [m, n, d], where each of the integer is allowed to changed from 1 to 10 independently, the prediction results of the best ARX model is shown in Figure 4.

However, for better performance, neurofuzzy modelling approach (ANFIS) was used to generate a nonlinear model by using a fuzzy inference sys- 

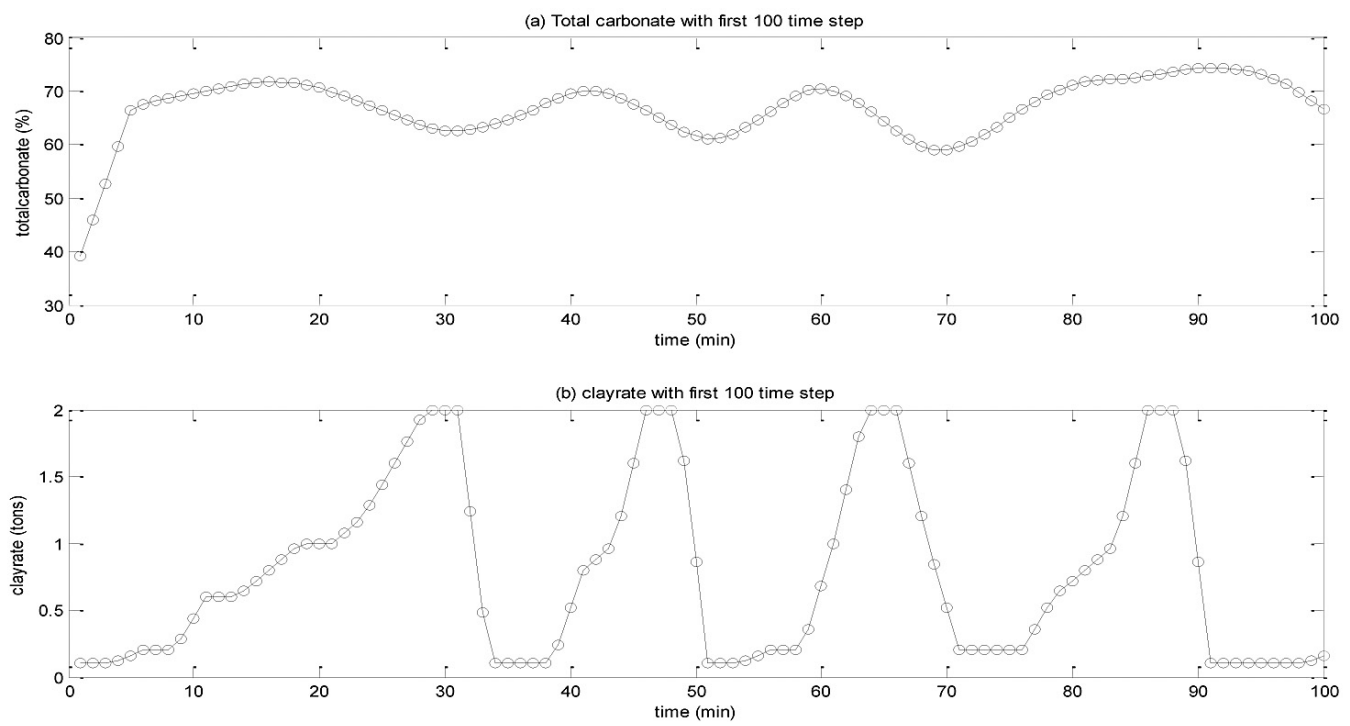

Fig. 3. Plot of the output $y(k)$ and input $u(k)$ for the first 100 time steps
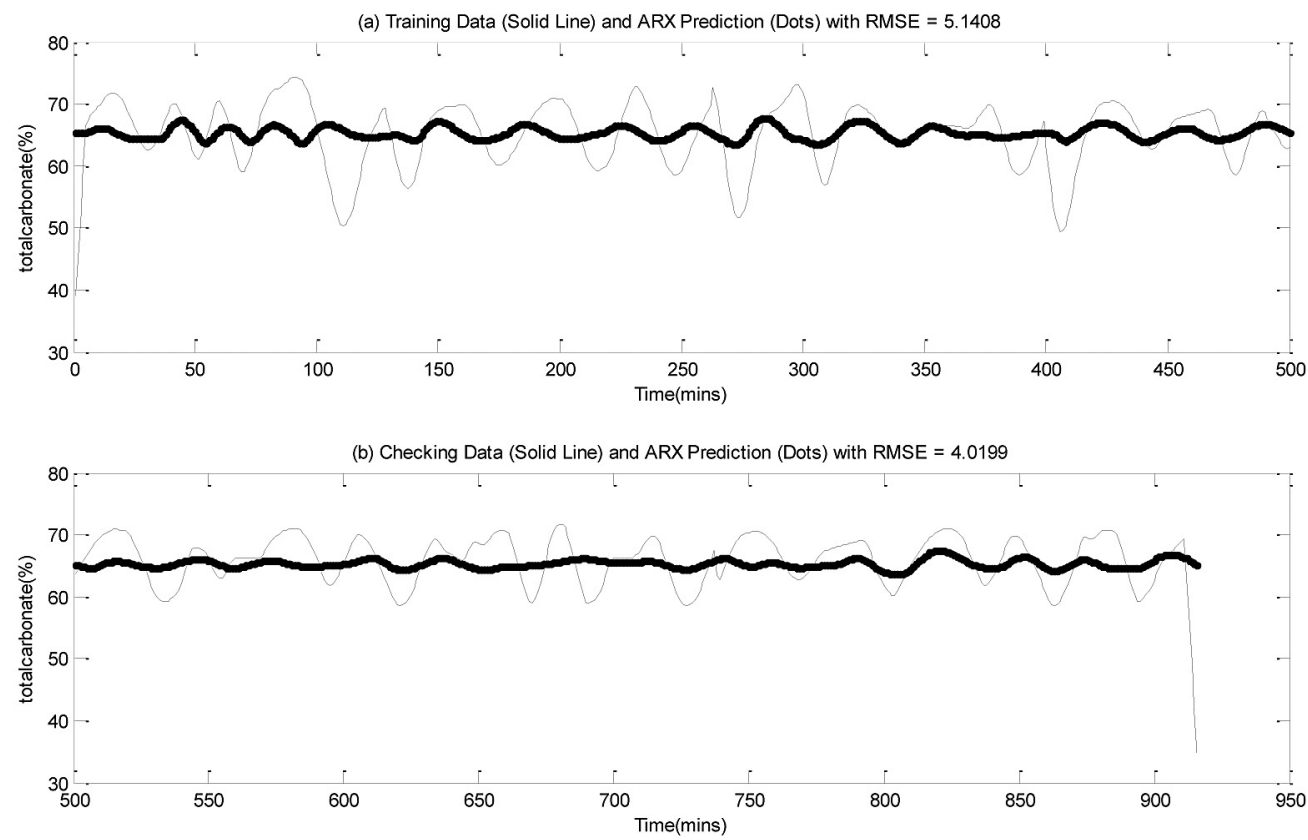

Fig. 4. ARX prediction with RMSE on training and checking data

tem. Input selection was carried out to determine which variable should be the input arguments to an ANFIS model. For simplicity, we suppose that there are 10 input candidates $(y(k-1), y(k-2), y(k-$ 3), $y(k-4), u(k-1), u(k-2), u(k-3), u(k-4), u(k-5)$, $u(k-6))$, and the output to be predicted is $y(k)$. A heuristic approach to input selection is called a sequential forward search, in which each input is selected sequentially as shown in Figure 5 to optimize the total squared error.

Model analysis was carried out to determine the performance of the model and to also determine if the model is fit for the data. In order to have a measure of the goodness of the identified models and predictors, Sum of Squares due to
Error (SSE). Correlation Coefficient (R square), Root Mean Squared Error (RMSE) and Fit were employed.

\section{RESULTS AND DISCUSSION}

Figure 4 shows a predictive performance of ARX model. It can be observed that the model gives a poor fit. The model is specified by model order $[\mathrm{m}, \mathrm{n}, \mathrm{d}]=[8,2,5]$, with a training RMSE of 5.1408 and a checking RMSE of 4.0199. The RMSE for both training and validation are too high indicating a poor prediction. The poor performance of ARX model may be due to nonlinear nature of 


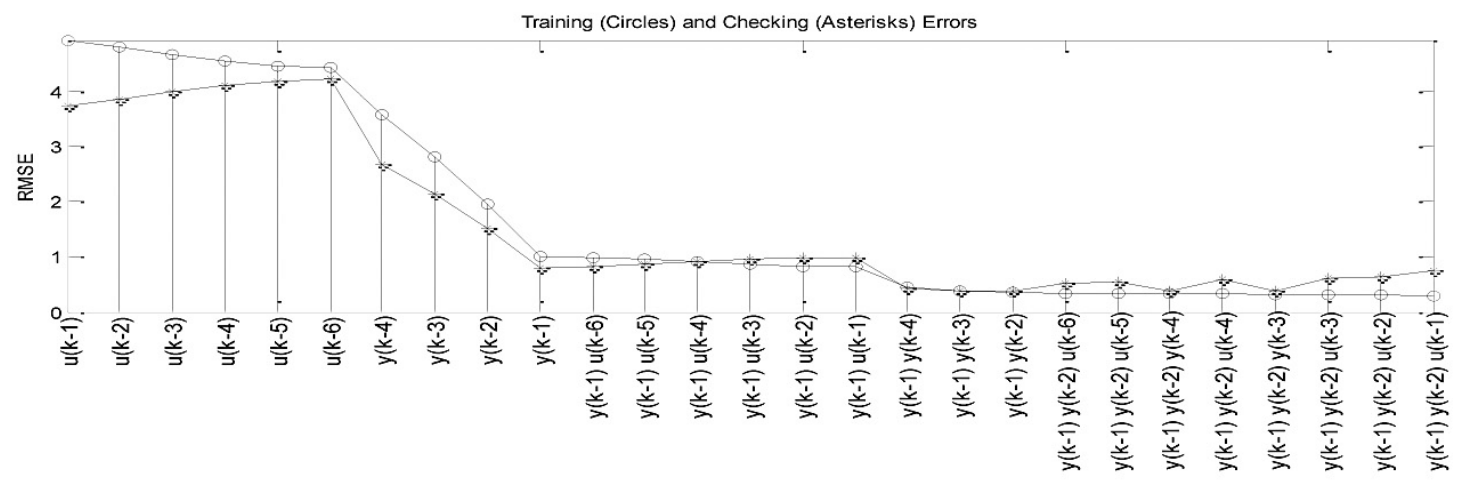

Fig. 5. Sequential ANFIS Input selection
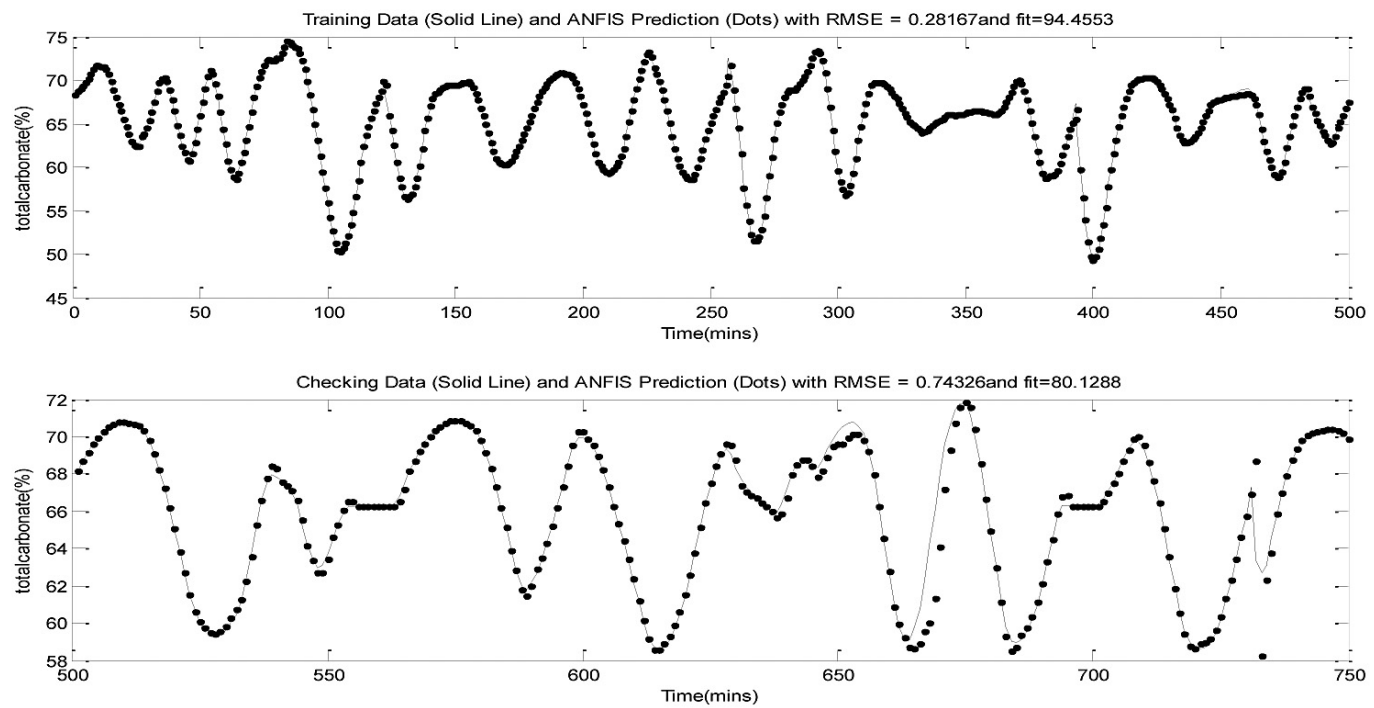

Fig. 6. ANFIS prediction with RMSE for training and checking data

the process which ARX cannot handle. Even if the performance is okay, the order of the model is too high $[8,2,5]$, for the model to be useful for control design. Inputs for ANFIS model were obtained based on sequential method, the selected inputs are $y(k-1), y(k-2)$ and $u(k-1)$, as shown in Figure 5. This is the point with the least values of RMSE for both training and checking data, obtained as 0.28167 and 0.74326 respectively.

Figure 6 shows ANFIS predictions on both training and checking data set. The network has 34 nodes and 8 rules. The results obtained for the performance indices gave SSE as 39.6692, R-Square as 0.9969, RMSE as 0.2817 and Fit of 94.4553 and 80.1288 for training and checking data respectively. A value close to 1 for the $\mathrm{R}$-square signifies that there is a strong correlation between each pair of data columns. Except for SSE which is very high, all other performance indices gave an indication of good performance. Figure 5 shows various combinations of input by the ANFIS model as searched sequentially.

\section{CONCLUSION}

Comparison between the two modelling approaches shows that ANFIS has satisfactorily modelled the behaviour of the blending process of cement plant while ARX approach gave a poor model. This may be attributed to nonlinear behaviour of the process.

\section{Acknowledgement}

The research input and output data was obtained by working in conjunction with Purechem Industries Limited, Onigbedu Village, Ogun State, Nigeria.

\section{REFERENCES}

1. Araromi D.O., Olu-Arotiowa O.A., Olajide J.O. and Afolabi T.J. Neuro fuzzy modelling approach for prediction of equilibrium moisture characteristics and shelf-life of corn flour. International Jour- 
nal of Soft Computing, 3(2), 2008, 159-166.

2. Araromi D.O., Afolabi T.J., Aloko D.F. Neural network control of CSTR for reversible reaction using reverence model approach. Leonardo Journal of Sciences, 6(10), 2007, 25-40.

3. Boskoski P., Stankovski M. Neuro-fuzzy controllers and application to autonomous robots. Journal of Mechanics, Automatic Control and Robotics, 7(1), 2008, 123-132.

4. Chi-Bin C. Process optimization by soft computing and its application to a wire bonding problem. Int. J. Appl. Sci. Eng., 2(1), 2004, 59.

5. Denis M., Keyvan N., Claude B., and Erwan G. An application of multivariate simulation in the cement industry. Mathemat. Geol., 37(5), 2005, 493-512.

6. George T.A. Shreve chemical processes. (Fifth Edition). McGraw-Hills, New York 1984.

7. Jin Y., Jiang J., Zhu J. Neural network based fuzzy identification and its application to modelling and control of complex systems. IEEE Trans. on Systems, Man and Cybernetics, 25(6), 1995, 990-997.

8. Kural A., Kizilaslan K., Ertugrul S., and Ozsoy C. A comparative study on modelling of raw material blending process in cement industry using conventional and intelligent technique. Control Application, 1, 2001, 736-741.

9. Lin C.J. and Xu Y.J. A hybrid evolutionary learning algorithm for TSK-type fuzzy model design, Mathematical and Computer Modelling, 43, 2006, 563-581.

10. Mat Darus I.Z. and Tokhi M.O. Modelling and control of a flexible structure using adaptive neuro-fuzzy inference system. In: Proceedings of the IEEE Conference on Mechatronics, ICM'2004, Istanbul, Turkey, 3-5 June, 2004.

11. Mat Darus I.Z. and Tokhi M.O. Adaptive neurofuzzy inference system modelling and control of a flexible plate structure. In: Proceedings of the 2003 UK Workshop on Computational Intelligence, UKCI'2003, Bristol, UK, 1-3 September, 2003.

12. Oni G. Personal contact. Purechem Industries Limited, Onigbedu Village, Ogun State, Nigeria 2010.

13. Ozsoy C., Kural A. and Baykara C. Modeling of the raw mixing process in cement industry. International Journal of Adaptive Control and Signal Processing, 18(5), 2004, 427-442. 